Riegel, über Auffindung und Bestimmung des Kupfers.

von Doppelcyaniden, wie sie vermuthet worden, fand nicht statt.

Aus diesen Versuchen darf ich wohl den Schluss ziehen, dass unter den gewöhnlichen Umständen die genannten Sauerstoffsalze keine chemische Verbindung mit Cyanquecksilber eingehen.

\title{
Ueber die Auffindung und Bestimmung des Kupfers;
}

von

\section{Dr. C. Rie gel in Carlsruhe.}

Die Auffindung des Kupfers in Verbindung mit anorganischen Substanzen bietet bekanntlich für den Geübten keine Schwierigkeiten, wohl aber bei Gegenwart von organischen, insbesondere nicht flüchtigen organischen Substanzen treten diese auf, indem dadurch das Verhalten des Kupfers resp. des Kupferoxyds sehr verändert wird.

Ausser den gewöhnlichen Kohlehydraten, wie die verschiedenen Zuckerarten, ist es vorzugsweise die Weinsteinsäure, welche bei den Kupferverbindungen (analog wie bei den Verbindungen des Eisens) die charakteristischen Reactionen derselben behinder $t$ oder gänzlich zweifelhaft macht. Ich erinnere hier an ein officinelles Präparat, den sogenannten Oxymel Aeruginis, das als eine Auflösung von neutralem essigsaurem Kupferoxyd in Verbindung mit Honig anzusehen ist. Durch den Honig oder vielmehr die darin enthaltene Zuckerart wird das Kupfersalz unter Entwicklung von Essigsäure desoxydirt, wodurch die grüne Farbe der Kupferauflösung in Rothbraun übergeht; ist das Präparat lange Zeit hindurch aufbewahrt worden, so findet man auch sehr fein zertheiltes metallisches Kupfer darin. Diese reducirende Eigenschaft der oben genannten Substanzen auf Kupferoxydsalze hat man auch als Gegengift gegen dieselben empfohlen und benutzt. Seil einer Reihe von Jahren bedient man sich auch dieses Verhaltens (unter Zusatz von Kali), um die Gegenwart des Zuckers nach- 
zuweisen, welche Methode vor der Pettenk ofer'schen (Anwendung von Schwefelsäure und Galle) den Vorzug verdient, und zwar besonders aus dem Grunde, weil man in neuerer Zeit öfter Gallenfarbstoff in dem Urin gefunden und dieser die schöne violette Färbung bei Gegenwart von Zucker und concentrirter Schwefelsäure erleidet und gerade zur Auffindung des Zuckers im Urin diese Methoden empfohlen wurden.

Durch die Einwirkung obengedachter Substanzen kann das Verbalten des kaustischen Kalis gegen Kupferoxyd wesentliche Modificationen erleiden. Ist die Menge des Kupfers nicht ganz unbedeutend, so entsteht zwar eine blaue Färbung, aber kein Niederschlag; beim Kochen wird das Kupferoxyd in den meisten Fällen reducirt, und es entsteht dann ein Niederschlag von Kupferoxydulhydrat, der gewöhnlich gelbbraun, oft rothbraun ist, und bisweilen wird das Kupfer fast gänzlich als Oxydul gefällt. In einigen organischen Substanzen, wie rothem Wein und ähnlich gefärblen, Weinsteinsäure enthaltenden Fliissigkeiten, welche viel Kupferoxyd enthalten, entsteht durch Kali (im Ueberschuss) eine schmutziggrüne Auflösung und ein ähnlich gefärbter Niederschlag, der durch Kochen rothbraun wird. Wenn diese Flüssigkeiten nur Spuren von Kupfer enthalten, so entsteht weder eine blaue Fürbung, noch beim Kochen ein Niederschlag. Ebenso hindert die Gegenwart nicht flüchtiger organischer Substanzen bisweilen die Erzeugung der blauen Farbe durch uberschuissiges Ammoniak in kupferhaltigen Flüssigkeiten, besonders wenn die Farbe derselben dunkel ist. In kupferhaltigem weissem Weine entsteht (hei nicht zu grossem Kupfergehalt) durch uberschüssiges Aetzammoniak eine schmutziggrüe oder braune Färbung, im rothen Weine eine schmutzigbraune Färbung.

Nach den Versuchen von Tuetz und Wackenroder wirken Aepfel- und Essigsäure auflösend auf Argentan und andere kupferhaltige Legirungen, selbst das gewöhnliche Silber, und eine längst bekannte Thatsache ist es, dass Substanzen, welche freie organische Säuren, insbe- 
sondere Essigsäure enthalten, in kupfernen Gefässen ohne Nachtheil, resp. Kupferaufnahme gekocht werden können, allein nicht darin erkalten dürfen. Meine vor einer Reihe von Jahren über die Anwendung des Kupfers im Haushalt angestellten sehr zahlreichen Versuche, welche zu dem mit obigem übereinstimmenden Resultate führten, dass organische Säuren im Verhältniss auflösender auf das Kupfer wirken, als die anorganischen, wurden dermalen behufs dev Auffindung des Kupfers zum grössern Theile wiederbolt. Man behandelte verschiedene Fruchtsäfte, saure Aepfel, saure Speisen der verschiedensten Art in kupfernen Gefässen längere Zeit in der Kochhilze und liess sie darin erkalten und in erkaltetem Zustande noch stehen. Kaffee mit und ohne Milch, Milch für sich, dunkles Bier, Traubenund Aepfelmost, Trauben-, Aepfel- und Birnmuss versetzte man mit Spuren von Kupfersalzlösungen. Auch hier zeigte sich die Reaction mit Kali und Ammoniak unzuverlässig, in vielen Fällen auch, besonders bei dunkelgefärbten Flüssigkeiten, Schwefelwasserstoffgas. In mehreren Fällen entstand durch letztgenanntes Reagens keine Fällung von Schwefelkupfer, während Kaliumeisencyanür und metallisches Eisen noch die Gegenwart des Kupfer's anzeigten. In solchen Auflösungen erfolgt durch Schwefelwasserstoffgas oder Schwefelwasserstoffammoniak meist keine sch warze, sondern eine braune Färbung, und die Abscheidung des Schwefelkupfers geht bisweilen äusserst langsam vor sich. Man darf nie unterlassen, in diesem Falle den entstandenen Niederschlag durch Auflösen in Säuren u. s. w. oder vor dem Löthrohr auf Kupfer zu prüfen.

Die empfindlichsten Reagentien für Kupfer sind Ferrocyankalium und blankes metallisches Eisen; ersteres eignet sich vorzüglich zur schnellen Entdeckung und selbst bei Gegenwart von einer bedeutenden Menge nicht flüchtiger organischer Substanzen. Die Aullösungen dürfen jedoch nicht zu dunkel gefärbt, müssen entweder neutral oder etwas sauer sein, wenn der charakteristische rothbraune Niederschlag entstehen soll. In den oben erwähn. ten Flüssigkeiten entstand, der grössern Anzahl der Ver- 
suche nach, wenn auch erst nach einiger Zeit, die Reaction mit dem Blutlaugensalz; Milch, welche nur äusserst geringe Spuren von Kupfersalz enthielt, nahm sogleich eine blassrothe Farbe an. In dunkelgefärbten Flussigkeiten, wie rothem Wein, Caffee mit etwas Milch versetzt, Extractlösungen, kann die Gegenwart des Kupfers nur dann mit Gewissheit durch Ferrocyankalium erkannt werden, wenn die Menge desselben nicht zu unbedeutend ist. In solchen Fluissigkeiten entdeckt man die Anwesenheit des Kupfers am besten durch ein blankes polirtes Eisen, wodurch das Kupfer im metallichem Zustand auf der Eisenfläche gefällt wird. Dieses Verfahren hat jedoch den Nachtheil, dass wenn die Menge des in der Substanz enthaltenen Kupfers sehr gering ist, der Ueberzug von metallischem Kupfer auf dem Eisen erst nach mehreren Stunden, oft selbst nach 24 Stunden erst erscheint, und dass die Fluissigkeit nicht zu sauer sein darf. Die Grenzen der Reactionen von Ferrocyankalium und von metallischem Eisen auf Kupfer scheinen ziemlich nahe bei einander zu liegen, ich sage scheinen, weil meinen Versuchen diese Absicht nicht zu Grunde lag.

In breiartigen oder festen organischen Substanzen lässt sich auf eine der angegebenen Verfahrungsarten das Kupfer nicht nachweisen; man erreicht jedoch seinen Zweck, wenn die Menge des letztern nicht ganz unbedeutend ist. durch Behandlung der Substanz mit Salpetersäure und Prüfung der filtrirten Flüssigkeit mit Ferrocyankalium u.s.w. Bei auusserst geringen Mengen von Kupfer gegen sehr viele organische Substanzen, wie bei Speisen, Brod (leider kommt eine Verfälschung desselben mit Kupfervitriol in Belgien und Frankreich nicht selten vor) u. s. w. bedient man sich am zweckmässigsten des Verfabrens von Jacquem yns, indem man die Substanz mit so viel Wasser behandelt, dass dadurch ein weicher Teig entsteht, dem man das Doppelte des Gewichts von gepulvertem kohlensaurem Kali oder (besser) Natron zumengt, das Gemenge in einem hessischen Tiegel nach und nach erhitzt und zuletzt eine Viertelstunde der Rothglühhitze aussetzt. Nach dem Erkalten 
wird die verkohlte Masse gepulvert, mit Wasser befeuchtet, in einem Achatmörser sehr fein zerrieben und mit mehr Wasser abgeschlämmt, bis die Kohle vollkommen abgeschieden und im Mörser metallisch glänzende kupferfarbene Schuppen zurïckbleiben, die nach irgend einem Verfahren noch näher zu untersuchen sind; diese Methode hat den Vorzug vor der von $\mathbf{K u h l m a n n}$ zur Auffindung kleiner Mengen von Kupferoxyd in mit einem Kupferoxydsalze vergifteten Brode, welches Verfahren darin besteht, dass eine ziemliche Menge ( $200 \mathrm{Gramme}$ ) der Substanz in einer Platinschale zu Asche verbrannt, diese mit Salpetersäure zu einem Brei vermischt, darauf zum Verdunsten des grössten Theils der freien Säure erhitzt, die Masse in Wasser aufgelöst und filtrirt wird. In dem Filtrate erkennt man leicht die Anwesenheit des Kupfers durch Schwefelwasserstoffgas und Ferrocyankalium.

Verguin in Lyon fand bei Gelegenheit der Analyse eines Kupfererzes, als er das Kupfer aus der Auflösung in einer Platinschale mittelst Eisens reduciren wollte, dass, sobald das Eisen mit dem Platin in Berührung kam, die. Platinschale mit einer sehr fest anhängenden Kupferlage überzogen wurde, ohne dass Kupfer auf das Eisen sich ansetzte; diese Kupferlage liess sich nur durch Behandlung mit Säure vom Platin trennen. Bei der Fällung des Kupfers durch Eisen muss die Lösung etwas sauer reagiren, jedoch darf sie nicht zu sauer sein und zu wenig Kupfer enthalten, indem sonst das Eisen schwarz und das Kupler nicht gehörig erkannt werden kann. Diesen Nachtheil äussert das Platin nicht, auch haftet das Kupfer an demselben fester. Aus diesen Gründen hat Verguin die Anwendung des Platins als Reagens auf Kupfer, besonders in gerichtlichen Fällen, empfohlen. Die Lösung darf bei diesem Verfahren nicht zu verdünt und muss mit Chlorwasserstoffsäure angesäuert sein; man giebt einen Tropfen der Lösung auf Platinblech, legt eine polirte Eisenklinge so darauf, dass das Eisen mit der Flüssigkeit und dem Platin in Beribrung tritt. Nach wenigen Secunden 
ist das Platin mit einer dichten, fest anhängenden Kupferschicht überzogen.

Von der Zweckmässigkeit dieses Verfahrens habe ich mich durch mehrere Versuche überzeugt mit thierischen Substanzen, die mit geringen Mengen eines Kupfersalzes intoxicirt worden, nach der oben beschriebenen Methode mit kohlensaurem Alkali sowohl, als auch nach dem unten folgenden Verfahren von Danger und Flandin. Diese empfehlen das Verfahren der Verkohlung, das sie bei Arsenvergiftungen vorgeschlagen, mit einigen Modificationen auch bei Vergiftungen mit Kupfer. Die thierische Substanz wird mit $\frac{1}{3}$ ihres Gewichts Schwefelsäure nach der bekannten Methode verkohlt, die Kohle bis zur Rothglühhitze erhitzt, gepulvert, mit Schwefelsäure befeuchtet und dann mit destillirtem Wasser behandelt. Aus den Versuchen von Danger und Flandin geht hervor, dass der menschliche Körper im normalen Zustande eben so wenig Kupfer als Arsen enthalte, so wie dass die Aussonderung des Kupfers nicht durch die Nieren, wie bei Antimon und Arsen, sondern durch die Lunge statt findet. Nach dem Tode findet man in den Intestinalröhren und der Leber das durch Absorption fortgerissene Kupfer; es ist deshalb bei gerichtlichen Untersuchungen auf diese Organe besondere Rücksicht zu nehmen. Danger und F land in bemerkten noch, dass durch einen längere Zeit hindurch dauernden Aufenthalt in Wasser der vergiftete Leichnam nicht alles Kupfer, welches er enthält, verliert.

In neuester Zeit ist von Bertozzi, Heller und E. v. Gorup-Besanez (conform mit den frühern Wabrnehmungen Duvergier's) in den Gallensteinen und von letzterem und Bramson selbst in der gesunden Menschengalle Kupfer aufgefunden worden. v. Gorup-Besanez äscherte menschliche Gallensteine mit Hülfe von Salpetersäure ein und fand in der zu einer lichtblauen Masse geschmolzenen Asche durch Behandlung mit verdünter Chlorwasserstoffsäure, Schwefelwasserstoff, Forrocyankalium und Eisen die Gegenwart von Kupfer und Eisen. 
Mit Recht empfieh]t der genannte Chemiker, um sehr geringe Mengen von Kupfer nachzuweisen, die Anwendung des Platins mit Zink statt des gewöhnlich benutzten Eisens, da dieses durch die freie Säure angegriffen wird und so bisweilen die Reaction nicht hinreichend deullich zeigt. In Rindsgalle fand v. Gorup-Besanez Spuren èines durch Schwefelwasserstoff fällbaren Metalls, welches Blei zu sein schien.

Bei der Darstellung der Gallensäure und deren Natronverbindung hatte ich Gelegenheit, mehrere Ochsengallen nach dem Verfahren des letztgenannten Chemikers auf einen Metall-, resp. Kupfergehalt zu prüfen; nur in einem Falle erhielt ich Spuren eines durch Schwefelwasserstoff fällbaren Metalls, ohne dasselbe genau bestimmen zu können. Da das Kupfer nicht bloss in vielen Küchengeschirren, sondern auch in manchen Pflanzen enthalten ist, so lässt sich wohl eine Quelle des Kupfers in der Galle finden, zumal wenn man damit die Beobachtung Walchner's über die stete Begleitung des Eisens durch Kupfer und Arsen verknuipft.

Ehe ich zur quantitativen Bestimmung des Kupfers schreite, habe ich noch des Verhaltens der schwefligen Säure gegen Kupfersalze Erwährung zu thun. Nach Berthier erleiden die Kupfersalze durch schweflige Säure keine Reduction, während aus den Versuchen von Vogel (Vater) hervorzugehen scheint, dass alle Kupferoxydsalze durch schweflige Säure in Oxydul verwandelt werden, und dass die Abscheidung nur bei denjenigen Salzen erfolgt, in welchen das Oxyd an keine energische Säure gebunden ist. Dieser Erfahrungen Vo gel's, die ich durch Versuche mit einigen Kupfersalzen besläligt fand, hat man sich in betreffenden Fällen zu erinnern, um nicht irregeleitet zu werden.

J on as hat die Darstellung der Phosphorsäure mittelst salpetersauren Kiupferoxyds empfohlen, und um die Zweckmässigket dieses Verfahrens (das jedenfalls der einfachen Oxydation des Phosphors mit reiner Salpetersäure nachsteht! zu pruffen, wurde in meinem Laboratorium diese 
Bereitungsweise versucht. Es wurde salpetersaures Kupferoxyd in 4-6 Theilen Wasser gelöst, die Lösung bis auf $70^{\circ}$ erhitzt und quentchenschwere Stuicke Phosphors hineingeworfen; mit dem Schmelzpunct des Phosphors trat Reaction ein, nämlich Reduction des Kupferoxyds und Oxydation des Phosphors, und zwar unter Abscheidung des ganzen Stickstoffgehalts als Gas, und nicht als Stickoxyd. Mit dem Phosphorzusatze hörte man auf, sobald das Ganze ein braunschwarzes Ansehen angenommen hatte. Nach Jonas soll man an einer abfiltrirten Probe sich durch Salmiakgeist überzeugen, ob der Kupfergehalt entfernt ist; da man selten den Punct trifft, wo alles Kupfer abgeschieden ist, so soll die Operation am zweckmässigsten unterbrochen werden, wenn die Flüssigkeit bis auf einen kleinen Schein von grüner Farbe alles salpetersaure Salz zersetzt zeigt, um solche entweder nochmals mit Phosphor zu schütteln, indem in einem Glase Säure und Phosphor erwärmt werden, oder selbige mittelst Schwefelwasserstoffgas zu reinigen. Im erstern Falle enthält, wenn auch ein noch so grosser Ueberschuss von Phosphor angewandt worden, die Säure stets noch Spuren von Kupfer, welche durch eine der oben beruhrten empfindlichen Prüfungsmelhoden aufgefunden werden können. Es bietet demnach die Jonas'sche Methode durchaus keinen Vortheil dar. Da ich, ähnlich den Versuchen von Vogel, mich mit der Einwirkung der phosphorigen Säure auf Kupfersalze beschäftigte, und zu ähnlichen Resultaten wie bei der Einwirkung der schwefligen Säure gelangte, konnte ich mir es nicht versagen, auch das Verhalten der unterphosphorigen Säure kennen zu lernen. Wurtz beobachtete nämlich bei der Einwirkung der letztern Säure auf Kupfersalze die Bildung von Kupferwasserstoff. Löst man einen Theil unterphosphorigsauren Baryt in Wasser, fällt die Lösung vollständig durch Schwefelsäure, fügt zur filtrirten Lösung $\stackrel{8}{T_{0}^{\circ}}$ Theile concentrirter schwefelsaurer Kupferlösung, erhitzt vorsichtig bis zu 70, so entsteht ein grünlicher, rothbraun werdender Niederschlag, welcher nach Wurtz aus 98,7 Kupfer und 1,22 
Wasserstoff bestehen soll, in der That aber nichts anderes. als reines Kupfer ist.

Was die quantitative Bestimmung des Kupfers und seiner Oxydationsstufen betrifft, so lässt sich das metallische Kupfer in seinen Verbindungen in Salpetersäure oder auch in Königswasser auflösen; in dieser Auflösung ist immer Kupferoxyd enthalten, das auf eine der folgenden Methoden gefällt, und aus dessen Gewicht man die Menge des metallischen Kupfers berechnet. Aehnlich ist die Bestimmung des Kupferoxyduls; ist dieses in einer Substanz enthalten, so wird dasselbe durch Auflösen in Salpetersäure in Kupferoxyd verwandelt, das durch Kali gefallt und aus dessen Gewicht nun die Menge des in der Substanz enthaltenen Kupferoxyduls berechnet wird.

Die gebräuchlichste Methode zur Bestimmung des Kupferoxyduls besteht in der Fällung desselben aus seinen Auflossungen durch reine Kalilösung, mit der Vorsicht, dass man die kupferoxydhaltige Flusssigkeit in einer Porcellan- oder Platinschale vorher vorsichtig zum Kochen erhitzt und dann mit einer Auflösung von Kali versetzt. Das gefälte braunschwarze Kupferoxyd wird vollkommen mit heissem Wasser ausgesüsst, getrocknet, geglüht und unmittelbar nach dem Erkalten im bedeckten Tiegel gewogen. Eine etwaige Reduction des Oxyds zu Oxydul lässt sich durch den spätern Luftstrom, den man beim Glühen im Tiegel erregt, beseitigen.

Wird die kupferoxydhaltige Flüssigkeit vor der Fallung mit Kali nicht zum Kochen erhitzt, so erhält man auf Zusatz des letztern einen voluminösen blauen Niederschlag von Kupferoxydhydrat, welcher eben des vollständigen Aussuissens und der genauen Beslimmung wegen immer durch Erhitzen in Kupferoxyd verwandelt werden muss. Auch hat man noch die Vorsicht zu gebrauchen, die kupferoxydhaltige Flüssigkeit, wenn dieselbe concentrirt ist, vorher mit vielem. Wasser zu verdünnen, wodurch eine vollständigere Fällung bewirkt wird, indem in concentrirten Flüssigkeiten nach uberschuissig zugesetztem Kali auch Kupferoxyd aufgelöst bleibt, wodurch man sich durch 
Schwefelwasserstoffammoniak und die oben berihrten Reagentien uberzeugen kann.

Die Verdünnung mit Wasser bietet noch den Vortheil, dass sich beim langen Kochen der Kupferoxydauflösung mit der Kalilösung nichts an die Wände des Gefässes anlegt, während dieses bei concentrirten Lösungen häufig der Fall ist. Oft sitzt das Kupferoxyd so fest, dass es mechanisch nicht abgelöst werden kann, sondern man muss diese kleine Menge in einigen Tropfen verdiunnter Chlorwasserstoffsäure aullösen und dann wiederum fällen.

Diese Methode mit Kali ist sehr praktisch und bei Beobachtung der gedachten Vorsichtsmaassregeln in den allermeisten Fällen hinreichend genau und deshalb empfehlenswerth.

Die Bestimmung des Kupferoxyds durch Fällen des Kupfers im regulinischen Zustande mittelst blanken Eisenblechs giebt deshalb kein ganz genaues Resultat, weil das regulinische Kupfer sich während des Trocknens oxydirt und auch häufig mit Kohle gemengt ist, welche, indem das Eisen aufgelöst wird, sich ausscheidet.

In neuerer Zeit sind mehrere Methoden zur Bestimmung des Kupferoxyds empfohlen, welche hier Erwähnung finden sollen. Runge hat auf das Verbalten des in Chlorwasserstoffsäure aufgelösten Einfachchlorkupfers bei Behandlung mit Kupfer, wodurch dasselbe in Halbchlorkupfer verwandelt wird und sich dabei eben so viel Kupfer auflöst, als in der angewendeten Menge Einfachchlorkupfer enthalten ist, eine quantitative Bestimmung des Kupfers gegründet. Man löst das nach der oben erwähnten Methode gefallte Kupferoxydhydrat in uberschissiger Chlorwasserstoffsäure und kocht die Lösung mit einer gewogenen Menge Kupfers so lange, bis die braune Farbe in eine hellgelbe übergegangen und sich nicht melır ändert. Diese Nethode ]ässt sich in vielen Fällen anwenden, wobei der Gewichtsverlust des Kupfers genau die Menge Kupfers anzeigt, die in der untersuchten Menge Oxyds enthalten ist. Kupfersalze, mit Ausnahme des salpetersauren, lassen sich auf diese Weise untersuchen, jedoch 
muss man auf die Gegenwart des Eisenoxyds Rücksicht nehmen, indem sich das Anderthalbchloreisen dem Einfachchlorkupfer äbnlich verhält. Ebenso ist auf die Anwesenheit des Manganoxyds Rücksicht zu nehmen, dessen nachtheiliger Einfluss sich übrigens dadurch beseitigen lässt, dass man die Auflösung in Chlorwasserstoffsaüre so lange kochen lässt, als sich noch Chlor entwickelt. Uebrigens versteht sich von selbst, dass die Abhaltung der Luft dabei sehr in Betracht zu ziehen ist, weshalb man den Versuch am besten in einer langhalsigen Retorte vornimmt.

Die von Levol Journ. de Pharm. et de Chim. 1842. Juillet) empfohlene Methode zur Kupferbestimmung ist auf ein ähnliches Verhalten gegründet. Das Verfahren besteht darin, dass man die Kupferlösung mit Aetzammoniak fällt und dann noch mehr Aetzammoniak hinzufügt, als zur Auflösung des entstandenen Niederschlags erforderlich ist. Die Flasche, worin sich die Lösung befindet, füllt man möglichst voll mit ausgekochtem, destillirtem Wasser, legt ein blankes, genau gewogenes Kupferblech hinein und verscbliesst sie luftdicht. Nach einigen Tagen, wenn sich die Flüssigkeit vollständig entfärbt hat, nimmt man das Kupferblech heraus, wäscht, trocknet und wägt es. Der Gewichtsverlust desselben zeigt genau die Menge des Kupfers an, welche in der geprüften Flüssigkeit war, weil das Oxyd 1 At. Sauerstoff auf 1 At. Kupfer enthält, und folglich noch 1 At. Kupfer aufnehmen muss, um in Kupferoxydul verwandelt zu werden. Nach der Angabe von Levol erhält man gleich richtige Resultate, ob das Kupferoxyd in Schwefel-, Salpeter- oder Chlorwasserstoffsäure aufgelöst ist. Bei Untersuchung von Legirungen, welche ausser Kupfer noch Zinn und Antimon enthalten, lässt sich dieses Verfahren anwenden, sobald diese Metalle durch Salpetersäure oxydirt worden; wenn gleich dasselbe mebrere Tage erfordert, so lässt es doch mehrere Versuche zu gleicher Zeit zu. Vergleichende Versuche, mit Legirungen aus Zinn und Kupfer (Kanonenmetall) angestellt, in welchen das vom oxydirten Zinn abgegossene Kupferoxyd der salpetersauren Lösung theils mittelst Kali- 
lösung, theils nach der Runge'schen, theils nach der L evol'schen Methode bestimmt wurde, gaben für letzlere ganz befriedigende, und im Allgemeinen ziemlich übereinstimmende Resultate.

Später veröffentlichte $\mathrm{Pelouze}$ eine neue Methode der quantitativen Bestimmung des Kupfers, welcher ein ähnliches Verfahren zu Grunde liegt, wie Barreswill zur Bestimmung des Krümelzuckers angegeben hat. Man löst ein bestimmtes Gewicht von Kupfer in Salpetersäure und versetzt allmälig die Lösung mit einem Gemisch von Weinsäure und Aetzkali, kocht das Ganze und setzt eine verdünnte Lösung von Zinnchlorür während des Kochens zu. Dadurch wird das Kupfer als Oxydul gefällt, während Zinn, Zink, Blei, Arsen und Antimon, womit das Kupfer verunreinigt sein kann, in der alkalischen Flussigkeit gelöst bleiben. Das Ende der Operation lässt sich an der Entfärbung der Flüssigkeit erkennen. Noch besser bewährte sich Pelouze's folgendes Verfahren. Man lost $1 \mathrm{Grm}$. ziemlich reines Kupfer in 7-8 Cub-Centim. käuflicher Salpetersäure, verdünnt mit etwas Wasser und setzt überschüssige Aetzammoniakflüssigkeit hinzu; ferner löst man $110 \mathrm{Grm}$. Einfachschwefelnatrium in $1 \mathrm{Litr}$. Wasser, bringt die Lösung in ein nach Cubikcentimetern graduirtes Probeglas und trägt die Lösung in die kupferhallige, kochende Fluissigkeit allmälig hinein. Dann löst man in Salpetersäure oder Königswasser eine bestimmte Menge der zu untersuchenden Legirung, versetzt mil überschüssigem Ammoniak, kocht und fügt wieder so lange von der Lösung des Schwefelnatriums hinzu, bis die blaue Farbe der Kupferlösung verschwunden ist. Durch die erste Probe hat man gefunden, wie viel von der Lösung des Schwefelnatriums nöthig war, um $1 \mathrm{Grm}$. Kupfer zu fällen, man wird daher aus der letztern Probe, aus der verbrauchten Lösung des Schwefelnatriums leicht berechnen können, wie viel Kupfer in $1 \mathrm{Grm}$. der untersuchten Legirung enthalten war. Nach Pelouze betragen die Beobachtungsfehler nicht uber $5 \mathbf{- 6}$ pro Mille, auch ist die Gegenwart anderer Metalle dieser Methode nicht hin- 
derlich. Bei Untersuchungen von Gemengen von Kupfer, Zinn, Zink, Cadmium, Blei, Antimon, Eisen, Arsenik und Wismuth wurde der Kupfergehalt stets bis auf 0,002 bis 0,003 gefunden. Beim Zusammenbringen der Schwefelverbindungen dieser Metalle mit einer ammoniakalischen Lösung von salpetersaurem Kupferoxyd wird diese entfärbt, demnach können sie nicht in der kupferhaltigen Lösung bestehen. Dies findet jedoch nur bis zur Enifärbung der Kupferlösung statt; später, resp. bei längerm Stehen werden wieder einige dieser Schwefelmetalle gebildet, welche Bildung jedoch keinen Einfluss auf die Bestimmung des Kupfers ausübt, da die Beobachtung derselben nur bis zu der Entfärbung der Flüssigkeit dauert. Zink und Cadmium werden erst dann gefällt, wenn die Lösung bereits farblos geworden, und können vielleicht auf ähnliche Weise gefällt werden; Kobalt und Nickel können wegen der gefärbten Auflösung, die sie mil Ammoniak geben, auf die Beslimmungsweise influiren. Bei Gegenwart von Silber hat man nur darauf Ruicksicht zu nehmen, dasselbe aus der salpetersauren Auflösung der Legirung durch Chlorwasserstoffsäure zu fällen. Zinn, welches häufig in Kupferlegirungen enthalten ist, findet sich in der ammoniakalischen Kupferlösung als Zinnsäure; diese bleibt lange suspendirt und hält eine geringe Menge Schwefelkupfer, wodurch sie gefärbt wird, zurück, so dass es schwer ist, zu bestimmen, wann die Flüssigkeit wirklich farblos geworden. Diese Unannehmlichkeit lässt sich jedoch leicht beseitigen, wenn man der Kupferlösung noch vor der Behandlung mit Ammoniak etwas salpetersaures Bleioxyd zusetz1; das Bleioxyd wird durch Ammoniak in Verbindung mit der Zinnsäure gefällt, so dass sich die Flüssigkeit mit grosser Regelmässigkeit klärt. Der durch Einwirkung der Schwefelnatriumlösung auf die heisse Kupferlösung entstehende Niederschlag hesteht nicht, wie man allgemein angenommen, aus reinem Schwefelmetalle, sondern ist ein Oxysulfür von 5 Aeq. Schwefel und 1 Aeq. Oxyd, welche Verbindung man auch durch Kochen von Schwefelkupfer mit schwefelsaurem 
Kupferoxyd erhält. Das Ammoniak hat bei dieser Bestimmungsweise nicht bloss den Vortheil, dass es die Farbe des Kupfers intensiver macht, sondern es verhindert auch die Fällung des Kupfers durch unterschwefligsaure Salze, deren Gegenwart sich in Lösungen von Schwefelmetallen fast nie vermeiden lässt, ferner durch schwefligsaure und unterschwefligsaure Salze, und in gehöriger Menge selbst die durch ätzende und kohlensaure Alkalien. Die Veränderungen, welche die Lösung des Schwefelnatriums erleidet, treten nur sehr langsam ein und lassen sich dadurch beseitigen, dass, wenn man eine Bestimmung vornehmen will, vorher reines Kupfer aufzulösen und damit der Schwefelgehalt der Probeflüssigkeit zu bestimmen ist.

Später machte Pelouze folgende Aenderungen in seiner Methode bekannt. Fällt man eine Auflösung eines Kupfersalzes durch Schwefelnatrium bei gewöhnlicher Temperatur, so erhält man $\mathrm{CuS}$, entsprechend dem $\mathrm{CuO}$, in Verbindung mit HIO. Kochl man diesen Niederschlag mit einer verdünnten Kupferlösung, so wird dieselbe entfärbt, was bei Gegenwart von Ammoniak noch schneller statt findet. Ueberschreitet man dabei nicht eine Temperatur von $75-80^{\circ}$, so hat der Niederschlag die constante Zusammensetzung von $\mathrm{CuS}, \mathrm{CuO}$. Erwärmt man aber auf $90^{\circ}$ oder selbst $90^{\circ}-100^{\circ}$, so nimmt dieser Niederschlag noch beträchtliche Mengen von Kupferoxyd auf. Zugleich wird ein bedeutender Theil des überschüssigen Kupfer. salzes in Oxydul verwandelt und bleibt in der ammoniakalischen Flüssigkeit gelöst, so dass man nun auf Zusatz von Schwefelnatrium in der farblosen Flüssigkeit einen schwarzen Niederschlag erhält. Es ist deshalb zur genauen Bestimmung des Kupfergehalts einer Lösung durch Schwefelnatrium nöthig, entweder bei sehr niedriger oder sehr hoher Temperatur zu operiren. Pelouze scheint die Temperatur von $75^{\circ}$ die geeignetste.

Zur Darstellung des Schwefelnatriums leitel man in eine kaustische Natronlauge von $36^{\circ}$ Beaumé (Seifensiederlauge) einen Strom von Schwefelwasserstoffgas, wodurch nach einigen Stunden Schwefelnatrium sich in Krystallen 
absetzt, die man aus der Flüssigkeit nimmt und mit kaltem Wasser abwäscht. Man löst von denselben etwa $140 \mathrm{Grm}$. in 1 Liter Wasser auf, lässt die trübe Flüssigkeit sich absetzen und nimmt, nachdem sie klar geworden, mit dem Heber ab. Ich habe bei meinen Versuchen gefunden, dass eine Filtration eben so gut ist und schneller zum Ziele führt. Die Concentration soll nach Pelouze der Art sein, dass $1 \mathrm{Grm}$. reines, in 5-6 Cub. - Centim. Salpetersäure gelöstes, und mit $40-50 \mathrm{Grm}$. Wasser versetztes Kupfer etwa 29-31 Cub.-Centim. zur Fällung braucht. Diese Normalflussigkeit bleibt Monate lang farblos und wird durch Säuren kaum getrübt. Zur Bestimmung ihres Gehalts löst man reines Kupfer (am besten auf galvanoplastischem Wege niedergeschlagen) in $5-6$ Cub.-Centim. käuflicher Salpetersäure auf, entfernt durch Kochen die rothen Dämpfe und giesst 50 Cuh.-Centim. käufliches Ammoniak hinzu. Das Ganze wird zum schwachen Kochen erhitzt, die Schwefelnatriumlösung tropfenweise zugegossen mit der Vorsicht, dass die Fliissigkeit fortwährend im Kochen erhalten wird. Sobald die blaue Farbe der Flüssigkeil nicht mehr zu erkennen ist, hört man mit dem Kochen auf, lässt die Flüssigkeit sich klären, wäscht den Rand der Schale mit wenig Ammoniak ab und giesst, je nach der Intensität der blauen Farbe, grössere oder geringere Mengen von Schwefelnatrium zur kochenden Flüssigkeit, worauf man wieder absetzen lässt und so lange das Zufuigen wiederholt, als noch die Flüssigkeit blau gefärbt erscheint. Zu Ende der Operation darf die Temperatur $80^{\circ}$ nicht iubersteigen, was nur selten der Fall ist, da das verdampfende Ammoniak sehr viel Wärme bindet. Bei sehr kurzem Docht kann man 20 Minuten lang kochen, ohne diese Temperatur zu überschreiten. Gegen Ende der Operation darf man nur einen, höchstens zwei Tropfen der Lösung zufïgen. Man bemerkt sich die Anzahl der Theile, die man von der Normallösung zur Fällung brauchte. Bei Untersuchung von Legirung ist es gut, vorher den Kupfergehalt annähernd zu bestimmen, damit man zur Prüfung etwa $1 \mathrm{Grm}$. Kupfer anwendet. Findet man den Gehalt an Kupfer $=\frac{9}{10}$ (wie 
beim Kanonengut), so nimmt man bei der Probe etwa $\frac{10}{9}=1,1 \mathrm{Grm}$. Man wägt die Probe und löst sie in Salpetersäure auf; bleibt ein unlöslicher Rückstand, der die Gegenwart von Antimon oder Zinn anzeigt, so löst man ihn, wenn er beträchtlich ist, durch Zusatz von Chlorwasserstoffsäure auf; ausserdem muss man in diesem Falle Blei zusetzen, damit die Zinnsäure, die sonst sehr lange suspendirt bleibt, schneller niedergeschlagen wird. Nach dem Erkalten setzt man 50 Cub.-Centim. flüssiges Ammoniak zu, bringt zum Kochen und verfährt ganz wie oben angegeben.

Gesetzt, man habe 29,5 Cub.-Centim. der normalen Schwefelnatriumlösung gebraucht, wäbrend $1 \mathrm{Grm}$. reines Kupfer 30 Cub.-Centim. erfordert, so beträgt das Kupfer $\frac{29,5}{30}=0,983$. Wog nun die angewandte Probe 1,1 Grm., so ist in 1000 Theilen der Legirung enthalten:

$$
\frac{0,983 \cdot 1000}{1,1}=893 \text { Kupfer. }
$$

Es ist einleuchtend, dass man sich nach Belieben (oder nach den zur Verfügung stehenden Gefässen) Normalflussigkeit bereiten und deren Gehalt auf ähnliche Weise, wie oben erwähnt, feststellen kann. Bei Untersuchungen von Legirungen des Kupfers mit Zinn und Antimon habe jch jedesmal aus der Hälfte der salpetersauren Lösung das Zinn oder Antimon und aus der andern Hälfte nach $P$ elo uze das Kupfer bestimmt. Wenn gleich das Verfahren umständlicb und zeitraubend $\mathrm{zu}$ sein scheint, so erlangt man nach einigen Versuchen so viel Uebung, dass man in wenigen Stunden die Quantität der einzelnen Metalle in $5-6$ und noch mehr solcher Legirungen bestimmen kann, und zwar was den Kupfergehalt anbetrifft, sehr genau. Dasselbe Verfahren lässt sich häufig auf Kupfererze oder Vitriole anwenden, bei denen das Eisen indessen durch Kochen mit Salpetersäure vollständig in Oxyd verwandelt sein muss, indem sonst ein Theil des Kupferoxyds in Oxydul ubergefuhrt wird.

Jacquelain und Casaseca haben gleichzeitig ein 
Verfahren bekannt gemacht, das wenig Zeit und Apparate erfordert, nämlich nur eine feine Wage, drei Glasröhren von gleichem Durchmesser, von denen nur eine graduirt sein muss, ein nach Decilitre graduirtes Probegläschen, eine Pipette, Salpetersäure, Ammoniak, Aetzkali, kohlensaures Kali und destillirtes Wasser. Man löst das Mineral oder die Legirung in Salpetersäure auf, filtrirt einen Theil davon, nachdem man ihn vorher mit Ammoniak versetzt und in der graduirten Glasröhre sein Volumen bestimmt hat, und setzt hierauf eine zu bestimmende Menge Wassers hinzu so lange, bis die Farbe mit der der Normalfüssigkeit übereinstimmt. Die Menge des in der Flüssigkeit enthaltenen Kupfers ergiebt sich aus dem Volumen, bis zu welchem man diese verdünnen musste, um eine bestimmte Farbennüance zu erhalten. Nickel hindert die Bestimmung nicht, wenn es nicht in zu grosser Menge zugegen ist. Zur Bereitung der Probeflüssigkeit löst man 0,5 Grm. reines Kupfer in verdünnter Salpetersäure, setzt etwas überschüssige Aetzammoniakfliussigkeit und so viel destillirtes Wasser hinzu, dass die Flüssigkeit bei $10^{\circ}$ gerade 1 Liter beträgt. Man giesst hierauf 5 Cub.-Centim. in eine der Glasröhren und bezeichnet den Stand der Flüssigkeit mit einem Theilstriche und schmilzt sie dann oben zu, so dass dieselbe keinen Verlust durch Verdunstung erleiden kann. Um die Nüancen der Farbe bosser bestimmen zu können, hält man die zwei mil einander zu vergleichenden Röhren vor ein Blatt Papier und bringt dieses zwischen das Auge und zerstreutes Licht, oder betrachtet sie durch ein planes blaugefärbtes, in einem Schirme befindliches Glas von 2 Millim. Durchmesser. Hat man z. B. 2 Grm. einer Legirung von Kupfer und Zink, so löst man diese in Salpetersäure auf, fügt Ammoniak hinzu und giesst die Flüssigkeit in eine graduirte Röhre von 2 Deciliter Capacität. Hat die Lösung nicht dieselbe Fürbung, wie die Normalflüssigkeit, so setzl man so viel Wasser hinzu, bis die Farbe beider Flüssigkeiten ganz gleich ist, und bestimmt nun aus dem Volumen der erhaltenen Lösung die Menge des darin befindlichen Kupfers. 
Die Zuverlässigkeit dieses Verfahrens scheint für den ersten Augenblick sehr fraglich zu sein, indem die Bestimmung von der Beobachtung der Intensität der Farbe der Lösung abhängt, somit von der individuellen Beobachtungsgabe, also keine scharfen Gränzen bieten kann, welche aber durch den Grad der Verdünnung einigermassen zu erreichen sind. Vergleichende Versuche scheinen die Zweifel zu beseitigen und die Möglichkeit einer ziemlich genauen Bestimmung zuzulassen, obgleich nicht geläugnet werden kann, dass stets Bedenken gegen eine solche Bestimmungsweise erhoben werden können. Ich bin daher auch nicht geneigt derselben das Wort zu reden; übrigens genügt sie vollkommen da, wo es sich nicht um eine äusserst genaue Bestimmung handelt.

Dr. Rieckher hat im Jahrbuche für praktische Pharmacie Bd. XV. Heft I, ebenfalls ein Verfahren zur Bestimmung des Kupfers publicirt, welches besonders behufs der Bestimmung des Kupfers in der Analyse des Kanonenmetalls ausgedacht wurde. Ohne mich auf die gebotenen Raisonnements einzulassen, die zu diesem Verfahren Veranlassung gaben, soll dieses selbst nur gewürdigt werden. Ganz mit dem Verfasser übereinstimmend sagte mir dies Verhalten der salpetersauren Salze im Allgemeinen, sowie die angestellen Versuche, dass die Zerlegung des salpetersauren Kupferoxyds (und somit auch die Bestimmung des Kupfers als Kupferoxyd aus demselben) Fehlerquellen in sich schliesse, die unter Umständen sehr ungenaue Resultate zur Folge haben könnten. Bei zu starker Hitze entsteht beim geschmolzenen Salze ein Spritzen, wobei ein Verlust nicht $\mathrm{zu}$ vermeiden ist; übrigens lässt sich dieses Spritzen hier, sowie bei den meisten Nitraten, kaum vermeiden. Diese Unannehmlichkeit soll bei Anwendung von Platingefassen beinahe ausgeschlossen werden und ich erlaube mir zu behaupten, dass dieses aber auch nur bein ahe der Fall ist. Die Anwendung von Platingefässen bietet jedoch eine neue Inconvenienz, indem durch die grosse Wärmeleitungsfähigkeit des Metalls die geschmolzene Masse an den Wänden bis zum Rande hinaufsteigt, welcher 
Uebelstand kaum bei dem schwächsten Feuer wird gehoben werden können. Aus diesem Grunde werden Abdampfschalen und später Tiegel von Porcellan empfohlen. Die von dem Zinnoxyd abgegossene salpetersaure Kupferlösung wird in einem Porcellantiegel im Wasserbade eingedampft, bis die Masse trocken erscheint, bei welchem Zeitpuncte eine klein wenig höhere Temperatur mit Nutzen angewandt werden kann, welche Dr. Rieckher dadurch erreicht, dass er den kupfernen Wasserbehälter, anstatt mit Wasser gefüllt, leer uber eine Spirituslampe stellt; es entweichen Wasser und Salpetersäure, ohne dass die Masse schmilzt. Wenn dieses eintreten will, wird der Tiegel in ziemlicher Entfernung über eine grosee Lampe mit doppeltem Luftzug gebracht und das schwächste Feuer gegeben, so dass bloss der Boden des Tiegels warm gehalten wird. Das Salz schmilzt und zieht sich in Krusten bis zur halben Höhe des Tiegels hinauf, wo es fest wird. Bei diesem Zeitpunct ändert sich die Farbe der Masse in Dunkelgrün und geht bei behutsamer Steigerung der Hitze in Schwarz uber. Um aber die totale Austreibung der Salpetersäure zu bewirken, wird der Porcellantiegel in einen hessischen gestellt und beide wohlverschlossen einem mässigen Glühfeuer ausgesetzt; noch vor dem gänzlichen Erkalten wird der Porcellantiegel herausgenommen und wegen der hygroskopischen Eigenschaft des Kupferoxyds warm gewogen.

Nach dieser eben beschriebenen Methode habe ich, abgesehen davon, dass mir von vornherein das Verfahren keine grossen Vortheile zu bieten schien, mehrere Versuche und zwar vergleichend mit den früher crwälnten unternommen.

In Folgendem will ich diese Versuche übersichtlich zusammenstellen:

a) Nach dem Rieckher'schen Verfahren.

I. 30 Gran metallisches Kupfer gaben...... 37,40 Gr. Cu 0 .

II. $30 "$ " " $"$ ".... 37,45" "

III. $40 "$ einer Legirung aus $10 \mathrm{Th} . \mathrm{Cu}$ und $1 \mathrm{Th}$. St gaben.............45,28" " 
40 Riegel, über Auffindung u. Bestimmung des Kupfers.

b) Nach der Methode mittelst Kali und Erhitzen.

I. 30 Gran metallisches Kupfer gaben....... 37,52 Gr. Cu0.

II. $30 "$ " "...... . . 37,48" "

III. 40 " einer Legirung aus $10 \mathrm{Th} . \mathrm{Cu}$ u. $1 \mathrm{Th}$. St gaben 45,40 " "

c) Nach der Methode von Pelouze mittelst Schwefelnalrium.

I. 30 Gran metallisches Kupfer gaben . . 29,98 Gr. metallisches $\mathrm{Cu}$.

II. 30

III. 40 "einer Legirung aus $10 \mathrm{Th}$. $\mathrm{Cu}$ und

$1 \mathrm{Th}$. St gaben ....... " $36,34 "$ " "

d) Nach der Methode von Runge.

I. 30 Gran metallisches Kupfer gaben . . 29,80 Gr. metallisches $\mathrm{Cu}$.

1I. $30 "$ " ". 29,73" " " " " " " " "

III. 40 " einer Legirung aus $10 \mathrm{Th}$. Cu und

1 Th. St gaben ....... . 36,25" " "

e) Nach der Methode von Lerol.

I. 30 Gran metallisches Kupfer gaben . . 29,70 Gr. metallisches $\mathrm{Cu}$.

II. $30 "$ " " . . 29,84" " "

III. $40 "$ einer Legirung aus $10 \mathrm{Th} . \mathrm{Cu}$ und

1 Th. St. gaben. ...... . 36,30" " "

Aus dieser Zusammenstellung lässt sich einigermassen auf die Genauigkeit und Zuverlässigkeit der funf Methoden schliessen. Wenn wir mit Berzelius das Kupferoxyd aus

$$
1 \text { At. Kupfer . . . 79,283. }
$$

bestehend annehmen, so ergeben sich für die erhaltenen Resultate folgende Zahlen:
a) I. 80,217 .
II. 80,107 .
III. 80,300 .
b) I. 79,957 .
II. 80,045 .
III. 80,088 .

Aus den Zahlen, welche die nach den drei anderen Methoden erzielten Resultate lieferten, ergiebt sich ferner, dass die genauesten Resultate nach der verbesserten Methode von Pelouzc erzielt wurden. Dieselbe verdient desshalb auch noch den Vorzug, dass, wenn die Normalflussigkeit vorräthig gehalten wird, sehr viele und genaue Versuche in kürzester Zeit angestellt werden können.

In Bezug auf die Rieck her'sche Methode bleibt noch zu bemerken, dass sie in Bezug auf Genauigkeit und Zeitersparniss keine besonderen Vortheile bietet und dass ausser den gedachten Nachtheilen, entweder nicht stark 
Meier, chem. Untersuchung des rothen Sandelholzes. 41

genug erhitzt wird und noch unzersetztes Kupferoxydsalz beigemengt bleibt, in welchem Falle bedeutende Fehler entstehen können, oder aber bei zu starker Hitze das Kupferoxyd in neues Oxyd $\mathrm{Cu}^{5} \mathrm{O}^{3}$ von Favre und Mauméne (wenigstens theilweise) verwandelt werden kann, wodurch ebenfalls bedeutende Fehler entstehen können. $\mathrm{Ob}$ es andern Chemikern gelingen wird, noch genauere Resultate zu erzielen, wollen wir dahin gestellt sein lassen. Von den bis jetzt bekannten Methoden zur quantitativen Bestimmung des Kupfers glaube ich aber die verbesserte von Pelouze und jene längst gebräuchliche durch Fällen mit kaustischem Kali unter den beschriebenen Cautelen empfehlen zu dürfen.

\title{
Chemische Untersuchung des rothen Sandelholzes;
}

\author{
von \\ Leo Meier, \\ Apotheker in Creutzburg in Preussen.
}

(Fortsetzung von Bd. CV, Heft 3. pag. 2y4.)

Santaloxyd.

Ich habe für diesen Körper diesen Namen gewählt, weil die Einwirkung der Säuren auf ihn eine auffallendo zu nennen ist. Obgleich es mir nicht geglückt ist, damit Verbindungen darzustellen, in welchen die Säure so weit neutralisirt ist, dass sie nicht mehr das Lackmuspapier röthet, so scheint es doch keinem $Z$ weifel unterworfen, dass die Säuren mit ihm Verbindungen eingehen können, wie man dieses aus dem Verlaufe meiner Arbeit ersehen wird.

Um das Santaloxyd zu bereiten, zieht man das geraspelte Sandelholz mit Aether oder 80 Proc. haltendem Weingeist aus, dampft den erhaltenen Auszug bis zur Trockne, kocht den Rückstand zu wiederholten Malen mit Wasser aus, löst ihn wieder in Weingeist und fällt die Santalsüure durch in Weingeist gelösten Bleizucker. Aus der gelben Flussigkeit, aus welcher das santalsaure 